\title{
THE GOFFMAN-LIU AREA AND PLATEAU'S PROBLEM
}

\author{
DAVID BINDSCHADLER AND TOGO NISHIURA
}

\begin{abstract}
Goffman and Liu defined a lower semicontinuous area $A(f)$ for linearly continuous maps $f$ from the disk into $\mathbf{R}^{n}$, showed that $A(f)$ is the Lebesgue area when $f$ is continuous and thereby extended the notion of area to some discontinuous maps. With the aid of a simple retraction of the punctured disk onto its boundary, a canonical linearly continuous map $f_{\Gamma}$ of the disk is associated with each continuous closed curve $\Gamma$ defined on the boundary of the disk. When $\Gamma$ is a Jordan curve, it is shown that the discontinuous map $f_{\mathrm{r}}$ has the property that $A\left(f_{\Gamma}\right)=A(\sigma)$ where $\sigma$ is a continuous map of least area spanning $\Gamma$ from the classical Plateau problem. Finally, the corresponding least area problem in the class of linearly continuous maps is shown to be trivial, that is, the least area is zero in the class of linearly continuous maps.
\end{abstract}

In the present paper we discuss the relationship between a lower semicontinuous area defined by Goffman and Liu [5] for a class of possibly discontinuous maps and the classical Plateau problem of least area for a Jordan curve in space. We show each Fréchet curve defined on $B$ (the boundary of the closed unit disk $D$ in $\mathbf{R}^{2}$ ) corresponds to a family of discontinuous maps defined on $D$ of the type of Goffman and Liu and that the areas of these maps depend only on the Fréchet curve. We show, for a Jordan Fréchet curve, the area of the discontinuous map associated with it is equal to the least area of the continuous surfaces defined on $D$ whose boundary values on $B$ are representations of the Jordan Fréchet curve.

Finally, we show that the least area problem in the class of linearly continuous maps is trivial, namely that the least area is zero.

1. Preliminaries. We will have need throughout the paper for the number $\sup \{|F(\xi)| \mid \xi \in S\}$ associated with a set $S$ and a map $F$. We will denote this number by $\|F\|_{s}$.

By a closed curve we mean a continuous map $\Gamma: B \rightarrow \mathbf{R}^{n}$. As usual we say two closed curves $\Gamma_{1}$ and $\Gamma_{2}$ are Fréchet equivalent if there is a sequence of orientation preserving diffeomorphisms $h_{k}: B \rightarrow B$ such that

$$
\lim _{k \rightarrow \infty}\left\|\Gamma_{1}-\Gamma_{2} \circ h_{k}\right\|_{B}=0 .
$$

A Fréchet curve is an equivalence class of closed curves under the Fréchet equivalence and we denote a class by $[\Gamma]$. There is a natural distance $\rho$ associated with Fréchet curves and $\rho\left(\left[\Gamma_{1}\right],\left[\Gamma_{2}\right]\right) \leqslant\left\|\Gamma_{1}-\Gamma_{2}\right\|_{B}$ (see [6, 9.1]).

Received by the editors July 9, 1979 and, in revised form, March 11, 1980; presented to the Society, January 6, 1980.

AMS (MOS) subject classifications (1970). Primary 28A75, 49F25; Secondary 49F10.

Key words and phrases. Lebesgue area, Fréchet curve, Plateau problem, minimal area. 
A measurable map $f: D \rightarrow \mathbf{R}^{n}$ is said to be linearly continuous if, for almost every $x, f \mid L_{x}$ is continuous $\left(L_{x}=\{(x, y) \mid y \in \mathbf{R}\}\right)$ and, for almost every $y, f \mid L^{y}$ is continuous $\left(L^{y}=\{(x, y) \mid x \in \mathbf{R}\}\right)$. Goffman and Liu defined a metric $d$ on the class $\mathcal{L}$ of linearly continuous maps [5]. They showed that $\mathcal{L}$ is the completion of the class of smooth maps defined on $D$ with the metric $d$. Furthermore, they showed that there is a lower semicontinuous area function $A(f)$ on $\mathcal{L}$ which agrees with the usual Lebesgue area for continuous maps. With $\mu$ as Lebesgue measure on the line, the metric $d$ is defined as

$$
d(f, g)=\max \left\{d_{1}(f, g), d_{2}(f, g)\right\}
$$

where

$$
\begin{aligned}
& d_{1}(f, g)=\inf \left\{\varepsilon \mid \mu\left\{x \mid\|f-g\|_{L_{x}}>\varepsilon\right\}<\varepsilon\right\}, \\
& d_{2}(f, g)=\inf \left\{\varepsilon \mid \mu\left\{y \mid\|f-g\|_{L^{y}}>\varepsilon\right\}<\varepsilon\right\} .
\end{aligned}
$$

Convergence in the metric $d$ corresponds to uniform convergence on almost every $L_{x}$ and on almost every $L^{y}$.

There is a canonical map $f_{\Gamma}: D \rightarrow \mathbf{R}^{n}$ associated with each closed curve $\Gamma$ by means of the radial retraction $r: D \backslash\{0\} \rightarrow B$ given by $r(\xi)=|\xi|^{-1} \xi$. That is, suppose $\Gamma: B \rightarrow \mathbf{R}^{n}$ is a closed curve. Then $f_{\Gamma}$ is the linearly continuous map

$$
f_{\Gamma}(\xi)= \begin{cases}(\Gamma \circ r)(\xi), & \xi \neq 0, \\ 0, & \xi=0 .\end{cases}
$$

2. Elementary facts about $f_{\Gamma}$. In this section we develop some elementary relationships between $f_{\Gamma}$ and continuous maps $g: D \rightarrow \mathbf{R}^{n}$.

2.1. Proposition. Let $g: D \rightarrow \mathbf{R}^{n}$ be a continuous map and let $\Gamma=g \mid B$. For each positive integer $k$, define $g^{k}: D \rightarrow \mathbf{R}^{n}$ by

$$
g^{k}(\xi)= \begin{cases}g(k \xi), & |\xi|<k^{-1}, \\ g\left(|\xi|^{-1} \xi\right), & k^{-1}<|\xi|<1 .\end{cases}
$$

Then $g^{k}$ is continuous, $g^{k} \mid B=\Gamma, d\left(f_{\Gamma}, g^{k}\right) \leqslant 2 k^{-1}$ and $A(g)=A\left(g^{k}\right)$.

Proof. Clearly $g^{k}$ is continuous and $f_{\Gamma}(\xi)=g^{k}(\xi)$ for $k^{-1}<|\xi|<1$. Hence we need only show $A(g)=A\left(g^{k}\right)$. From [5], we have that $A(g)$ and $A\left(g^{k}\right)$ are the Lebesgue areas of $g$ and $g^{k}$. One easily sees that $g$ and $g^{k}$ are Fréchet equivalent maps. Hence $A(g)=A\left(g^{k}\right)$ follows from $[1, \S \S 6.3,31.7]$.

2.2. Proposition. Let $\Gamma$ be a closed curve. Then

$$
A\left(f_{\Gamma}\right)<\inf \{A(g) \mid g \text { is continuous and } g \mid B=\Gamma\} .
$$

Proof. Suppose $g$ is continuous with $g \mid B=\Gamma$. Let $g^{k}$ be as in Proposition 2.1 above. The lower semicontinuity of the area function gives $A\left(f_{\Gamma}\right)<\underline{\lim }_{k \rightarrow \infty} A\left(g^{k}\right)$ $=A(g)$. The proposition now follows.

2.3. Corollary. Let $\Gamma$ be a closed curve. If $g_{k}: D \rightarrow \mathbf{R}^{n}$ is a sequence of continuous maps such that $\lim _{k \rightarrow \infty}\left\|g_{k}-\Gamma\right\|_{B}=0$ then $A\left(f_{\Gamma}\right)<\underline{\lim }_{k \rightarrow \infty} A\left(g_{k}\right)$. 
Proof. Let $\Gamma_{k}=g_{k} \mid B$. Then $\lim _{k \rightarrow \infty} d\left(f_{\Gamma}, f_{\Gamma_{k}}\right)=0$, since $f_{\Gamma_{k}}$ converges to $f_{\Gamma}$ uniformly on each line $L_{x}$ and $L^{y}$. Hence $A\left(f_{\Gamma}\right)<\lim _{k \rightarrow \infty} A\left(f_{\Gamma_{k}}\right)<\underline{\lim }_{k \rightarrow \infty} A\left(g_{k}\right)$, the last inequality being a consequence of Proposition 2.2.

2.4. Proposition. Let $\Gamma$ be a closed curve and $0<\varepsilon<2 \sqrt{2}$. Then there is $\delta>0$ such that, for each continuous map $g$ with $d\left(f_{\Gamma}, g\right)<\delta$, there is a continuous map $\overline{\mathbf{g}}$ with $A(\bar{g})<A(g), d\left(f_{\Gamma}, \bar{g}\right)<\varepsilon$ and $\|\Gamma-\bar{g}\|_{B}<d\left(f_{\Gamma}, g\right)$.

Proof. Let $\delta=\varepsilon / 4$ and suppose $g$ is a continuous map with $d\left(f_{\Gamma}, g\right)<\delta$. Then $d_{1}\left(f_{\Gamma}, g\right)<\delta$ and $d_{2}\left(f_{\Gamma}, g\right)<\delta$, where $d_{1}$ and $d_{2}$ come from the definition of $d$. So there is a number $\eta \in(0, \varepsilon / 2)$ so that when $t= \pm \eta$ we have

$$
\left\|f_{\Gamma}-g\right\|_{L_{i}}<d_{1}\left(f_{\Gamma}, g\right)<\delta ; \quad\left\|f_{\Gamma}-g\right\|_{L^{\prime}}<d_{2}\left(f_{\Gamma}, g\right)<\delta .
$$

Denote by $C$ the boundary of $I=[-\eta, \eta] \times[-\eta, \eta]$. Since $\delta<\sqrt{2} / 2, C$ is interior to $D$ and for each $\xi \in D \backslash I$ there is a unique point $\zeta(\xi)$ in $C$ such that $\xi, \zeta(\xi)$ and 0 are colinear. We define $\bar{g}$ as

$$
\bar{g}(\xi)= \begin{cases}g(\xi), & \xi \in I, \\ g(\zeta(\xi)), & \xi \in D \backslash I .\end{cases}
$$

Clearly, $\bar{g}$ is continuous. So, $A(\bar{g})=A(\bar{g} \mid I)=A(g \mid I) \leqslant A(g)$. The definitions of $\bar{g}$ and $f_{\Gamma}$ give $\left\|f_{\Gamma}-\bar{g}\right\|_{D \backslash I}<\delta$. So, $d\left(f_{\Gamma}, \bar{g}\right)<\varepsilon$.

For $\xi \in B$ we have

$$
|\Gamma(\xi)-\bar{g}(\xi)|=\left|f_{\Gamma}(\zeta(\xi))-g(\zeta(\xi))\right| \leqslant\left\|f_{\Gamma}-g\right\|_{C}<\max \left[d_{1}\left(f_{\Gamma}, g\right), d_{2}\left(f_{\Gamma}, g\right)\right] .
$$

Consequently, $\|\Gamma-\bar{g}\|_{B}<d\left(f_{\Gamma}, g\right)$. The proposition is completely proved.

3. The area of $f_{\Gamma}$.

3.1. Theorem. Let $\Gamma$ be a closed curve. Then $A\left(f_{\Gamma}\right)=\min \left\{\underline{\lim }_{k \rightarrow \infty} A\left(g_{k}\right) \mid\left\{g_{k}\right\}\right.$ is a sequence of continuous maps such that $\lim _{k \rightarrow \infty}\left\|g_{k}-\Gamma\right\|_{B}=\overline{0\}}$.

Proof. There is a sequence of continuous maps $\left\{g_{k}\right\}$ such that $A\left(f_{\Gamma}\right)=$ $\lim _{k \rightarrow \infty} A\left(g_{k}\right)$ and $\lim _{k \rightarrow \infty} d\left(f_{\Gamma}, g_{k}\right)=0$. By Proposition 2.4, there is a subsequence, still denoted by $g_{k}$, such that associated with each $g_{k}$ is a continuous map $\bar{g}_{k}$ such that $A\left(\bar{g}_{k}\right) \leqslant A\left(g_{k}\right)$ and $\lim _{k \rightarrow \infty}\left\|\Gamma-\bar{g}_{k}\right\|_{B}=0$. Corollary 2.3 now implies $A\left(f_{\Gamma}\right)<\underline{\lim }_{k \rightarrow \infty} A\left(\bar{g}_{k}\right)<\varlimsup_{k \rightarrow \infty} A\left(\bar{g}_{k}\right)<\lim _{k \rightarrow \infty} A\left(g_{k}\right)=A\left(f_{\Gamma}\right)$. The theorem is proved.

3.2. Theorem. Let $[\Gamma]$ be a Fréchet curve. Then $A\left(f_{\Gamma}\right)=\min \left\{\underline{\lim }_{k \rightarrow \infty} A\left(g_{k}\right) \mid\left\{g_{k}\right\}\right.$ is a sequence of continuous maps such that $\left.\lim _{k \rightarrow \infty} \rho\left(\left[g_{k} \mid B\right],[\Gamma]\right)=0\right\}$.

Proof. Let $\left\{g_{k}\right\}$ be a sequence of continuous maps for which $\rho\left(\left[g_{k} \mid B\right],[\Gamma]\right) \rightarrow 0$ as $k \rightarrow+\infty$. There exists a sequence of homeomorphisms $\left\{h_{k}\right\}$ of $B$ such that $\left\|\left(g_{k} \mid B\right) \circ h_{k}-\Gamma\right\|_{B} \rightarrow 0$ as $k \rightarrow+\infty$. For each $h_{k}: B \rightarrow B$ define $H_{k}: D \rightarrow D$ by

$$
H_{k}(\xi)= \begin{cases}|\xi| h_{k}\left(|\xi|^{-1} \xi\right), & \xi \neq 0, \\ 0, & \xi=0 .\end{cases}
$$

Since $\left(g_{k} \mid B\right) \circ h_{k}=\left(g_{k} \circ H_{k}\right) \mid B$, we can use Theorem 3.1 and [1, §6.3] to conclude

$$
A\left(f_{\Gamma}\right) \leqslant \underline{\lim }_{k \rightarrow \infty} A\left(g_{k} \circ H_{k}\right)=\underline{\lim }_{k \rightarrow \infty} A\left(g_{k}\right) \text {. }
$$


So $A\left(f_{\Gamma}\right)<\inf \left\{\lim _{k \rightarrow \infty} A\left(g_{k}\right) \mid\left\{g_{k}\right\}\right.$ is a sequence of continuous maps such that $\left.\lim _{k \rightarrow \infty} \rho\left(\left[g_{k} \mid B\right],[\Gamma]\right)=0\right\}$. To complete the proof apply Theorem 3.1 again.

3.3. Corollary. Let $[\Gamma]$ be a Fréchet curve. Then

(1) $A\left(f_{\Gamma_{1}}\right)=A\left(f_{\Gamma_{2}}\right)$ whenever $\Gamma_{1}, \Gamma_{2} \in[\Gamma]$,

(2) $A\left(f_{\Gamma}\right)<\inf \{A(g) \mid g$ is continuous with $g \mid B \in[\Gamma]\}$.

If $[\Gamma]$ is a Jordan Fréchet curve in $\mathbf{R}^{n}$, then it is known ([3] and [8]) that there is a continuous surface $\sigma: D \rightarrow \mathbf{R}^{n}$ spanning $\Gamma$ of least area in the class of continuous surfaces spanning $\Gamma$. We prove below that the discontinuous map $f_{\Gamma}$ has GoffmanLiu area equal to the Lebesgue area of this continuous surface $\sigma$ of least area.

\subsection{Theorem. Suppose $\Gamma$ is a closed Jordan curve. Then}

$$
\begin{aligned}
A\left(f_{\Gamma}\right) & =\min \{A(g) \mid g \text { is continuous with } g \mid B \in[\Gamma]\} \\
& =\min \{A(g) \mid g \text { is continuous with } g \mid B=\Gamma\} .
\end{aligned}
$$

Proof. In view of Corollary 3.3(2) we may assume $A\left(f_{\Gamma}\right)<\infty$. The first equality now follows from Theorem 3.2 and [6, Theorem 9.4.1].

To prove the second equality let $\sigma$ denote a continuous surface of least area with $\sigma \mid B \in[\Gamma]$. In a straightforward manner one can define a continuous map $\bar{\sigma}$ : $D \rightarrow \mathbf{R}^{n}$ such that $\bar{\sigma} \mid B=\Gamma, \bar{\sigma}(\xi)=\sigma(2 \xi)$ whenever $0 \leqslant|\xi|<2^{-1}$ and $A(\bar{\sigma})=A(\sigma)$.

4. Least area problem for linearly continuous maps. The problem of least area in the class of linearly continuous maps is trivial as shown by the following theorem.

4.1. TheORem. For any continuous curve $\Gamma: B \rightarrow \mathbf{R}^{n}$,

$$
0=\min \{A(f) \mid f \text { is linearly continuous and } f \mid B=\Gamma\} .
$$

Proof. Consider the polar coordinates for the plane where the pole is a point on $B$ and the polar axis is the diameter of $D$ passing through the pole. Define the discontinuous map $p: D \rightarrow B$ by

$$
p(r, \theta)= \begin{cases}(2 \cos \theta, \theta), & \text { if } r \neq 0, \\ (0, \theta), & \text { if } r=0 .\end{cases}
$$

Note that the location of the pole and polar axis yields the facts that $p \mid B$ is the identity on $B$ and $p(D)=B$. If $\Gamma: B \rightarrow \mathbf{R}^{n}$ is any continuous curve, define $M_{\Gamma}$ : $D \rightarrow \mathbf{R}^{n}$ by the formula $M_{\Gamma}=\Gamma \circ p$. The map $M_{\Gamma}$ is linearly continuous and $M_{\Gamma} \mid B=\Gamma$. We will now demonstrate that $A\left(M_{\Gamma}\right)=0$.

Let $(X, Y)$ be the natural rectangular coordinates associated with the polar coordinates defined above. Thus, $X=r \cos \theta$ and $Y=r \sin \theta$. Then for each positive integer $k>1$, define $p_{k}: D \rightarrow D$ by

$$
p_{k}(X, Y)= \begin{cases}(X, Y), & \text { if } X>k^{-1}, \\ \left(k^{-1}, Y\right), & \text { if } X<k^{-1}\end{cases}
$$

Then the sequence $\left\{g_{k}\right\}$, where $g_{k}=\Gamma \circ p \circ p_{k}$, is a sequence of continuous maps such that $A\left(g_{k}\right)=0$ and $d\left(g_{k}, M_{\Gamma}\right) \leqslant 2\left(2 k^{-1}-k^{-2}\right)^{1 / 2}$. That $A\left(g_{k}\right)=0$ follows from the fact that $g_{k}: D \rightarrow \mathbf{R}^{n}$ can be factored through the Jordan $\operatorname{arc} p \circ p_{k}(D)$. 
The fact $A\left(M_{\Gamma}\right)=0$ now follows from the lower semicontinuity of the area function.

REMARKS. For further references to discussions of the class of linearly continuous maps $\mathcal{L}$ see [4] and [7].

The authors wish to acknowledge Professor H. Blaine Lawson, Jr. for some helpful conversations and the referee for suggesting that we examine the least area problem for linearly continuous maps.

\section{REFERENCES}

1. L. Cesari, Surface area, Ann. of Math. Studies, no. 35, Princeton Univ. Press, Princeton, N. J., 1956.

2. R. Courant, Dirichlet principle, conformal mappings, and minimal surfaces, Interscience, New York, 1950.

3. J. Douglas, Solution of the problem of Plateau, Trans. Amer. Math. Soc. 33 (1931), 263-321.

4. C. Goffman, Bounded variation and absolute continuity in the theory of surface area, Real Anal. Exchange 4 (1978-79), 9-47.

5. C. Goffman and F.-C. Liu, Discontinuous mappings and surface area, Proc. London Math. Soc. 20 (1970), 237-248.

6. C. Morrey, Multiple integrals in the calculus of variations, Springer-Verlag, New York, 1966.

7. T. Nishiura, Some examples relating surface area and the area integral, Real Anal. Exchange 4 (1978-79), 58-65.

8. T. Rado, On the problem of least area and the problem of Plateau, Math. Z. 32 (1930), 763-796.

Department of Mathematics, Wayne State University, Detroit, Michigan 48202 